
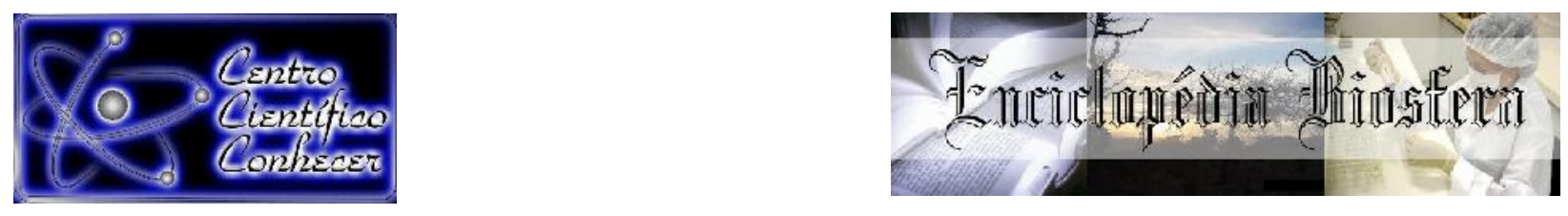

\title{
Bradyrhizobium sp., Azospirillum brasilense E ADUBAÇÃO FOSFATADA NA CULTURA DO FEIJÃO-CAUPI CULTIVADO EM AMBIENTE AMAZÔNICO
}

\author{
Érica de Oliveira Araújo ${ }^{1}$ \\ ${ }^{1}$ Prof.(a) Doutora do Departamento de Agropecuária do Instituto Federal de Educação, \\ Ciência e Tecnologia de Rondônia, Campus Colorado do Oeste - Brasil. \\ email:erica.araujo@ifro.edu.br
}

\section{Recebido em: 06/04/2019 - Aprovado em: 10/06/2019 - Publicado em: 30/06/2019 DOI: 10.18677/EnciBio_2019A43}

\begin{abstract}
RESUMO
Informação de respostas de genótipos de feijão-caupi quanto as particularidades relacionadas à promoção de crescimento pelo Azospirillum brasilense aliado a Bradyrhizobium sp. são escassas na literatura. Diante do exposto, o presente trabalho teve por objetivo avaliar os efeitos da inoculação com Bradyrhizobium sp. e Azospirillum brasilense em associação com a adubação fosfatada sobre os componentes morfológicos e de produção da cultura do feijão-caupi cultivada na região sul do Estado de Rondônia. O experimento foi realizado no período de fevereiro a maio de 2018, em área experimental, munícipio de Colorado do Oeste, RO, em um Latossolo Vermelho-amarelo. $\mathrm{O}$ delineamento experimental utilizado foi o de blocos casualizados, com três repetições, arranjados em esquema fatorial $3 \times 3$. O primeiro fator foi constituído por três doses de fósforo $\left(0,80\right.$ e $120 \mathrm{~kg} \mathrm{ha}^{-1}$ de $\left.\mathrm{P}_{2} \mathrm{O}_{5}\right)$; e o segundo fator, compreendeu ausência, inoculação e coinoculação com rizobactérias, perfazendo um total de 9 tratamentos, e 27 unidades experimentais. A dose de $120 \mathrm{~kg} \mathrm{ha}^{-1}$ de $\mathrm{P}_{2} \mathrm{O}_{5}$, na presença de Bradyrhizobium $s p$. é suficiente para promover o máximo crescimento e desenvolvimento de plantas de feijão-caupi cultivada em ambiente amazônico. A produtividade de grãos aumentou na ordem de $38 \%$ quando inoculado com Bradyrhizobium sp. e adubado com $120 \mathrm{~kg} \mathrm{ha}^{-1}$ de $\mathrm{P}_{2} \mathrm{O}_{5}$. A adição de $\mathrm{P}_{2} \mathrm{O}_{5}$ no solo promoveu aumento nas concentrações de $\mathrm{N}$ e $\mathrm{K}$ nas folhas de plantas de feijão-caupi em pleno florescimento. A coinoculação não influenciou os componentes morfológicos e produtivos da cultura do feijão-caupi, no entanto deve ser mais bem elucidada, visto que há possibilidades de interação entre os microrganismos, contribuindo para o alcance da sustentabilidade no setor agrícola.
\end{abstract}

PALAVRAS-CHAVE: Vigna unguiculata, produtividade, Rondonia.

\section{Bradyrhizobium sp., Azospirillum brasilense AND PHOSPHATE FERTILIZATION ON COWPEA CULTURE GROWN IN AMAZONIAN ENVIRONMENT}

\section{ABSTRACT}

Information from responses of genotypes of cowpea as the particularities related to growth promotion by Azospirillum brasilense combined with Bradyrhizobium sp. are scarce in the literature. On the above, the present study aimed to evaluate the effects of inoculation with Bradyrhizobium sp. and Azospirillum brasilense in association with 
phosphate fertilization on the morphological and production components of the cowpea crop cultivated in the southern region of the State of Rondônia. The experiment was conducted from february to may 2018, in the municipality of Colorado do Oeste, $\mathrm{RO}$, in a red-yellow latosol. The experimental design used was randomized blocks with three replications, arranged in a $3 \times 3$ factorial scheme. The first factor was made up of three rates $\left(0,80\right.$ and $120 \mathrm{~kg} \mathrm{ha}^{-1}$ of $\left.\mathrm{P}_{2} \mathrm{O}_{5}\right)$; and the second factor, understood the absence, inoculation and coinoculation with rhizobacteria, making a total of 9 treatments, and 27 experimental units. The rates of $120 \mathrm{~kg} \mathrm{ha}^{-1}$ of $\mathrm{P}_{2} \mathrm{O}_{5}$, in the presence of Bradyrhizobium $s p$. is enough to promote maximum growth and development of plants of cowpea cultivated in amazonian soils. Grain productivity increased in the order of $38 \%$ when inoculated with Bradyrhizobium sp. and composted with $120 \mathrm{~kg} \mathrm{ha}^{-1}$ of $\mathrm{P}_{2} \mathrm{O}_{5}$. The addition of $\mathrm{P}_{2} \mathrm{O}_{5}$ in soil promoted increase in concentrations of $\mathrm{N}$ and $\mathrm{K}$ in the leaves of cowpea plants in full bloom. The coinoculação did not influence the morphological and productive components of the culture of cowpea, however must be understood, since there are possibilities of interaction between microorganisms, thus contributing to the achievement of sustainability in agricultural sector.

KEYWORDS: Vigna unguiculata, productivity, Rondonia.

\section{INTRODUÇÃO}

O feijão-caupi (Vigna unguiculata (L.) Walp.) é nativo da África e bastante cultivado nas regiões tropicais dos continentes africano, asiático e americano (SILVA et al., 2008). Estima-se que $70 \%$ do feijão produzido no território brasileiro sejam do feijão comum (Phaseolus vulgaris) e $30 \%$ do feijão-caupi (COUTINHO et al., 2014). Contudo, esses dados não descrevem a realidade dos Estados das regiões Norte e Nordeste, cuja produção é quase que exclusiva de feijão-caupi, no qual juntas são responsáveis por 1.192 milhões de hectares cultivados na safra 2017/18 (CONAB, 2018). Porém, baixos níveis de produtividade têm sido observados nas áreas produtoras e uma das principais causas é a baixa disponibilidade de nutrientes no solo, particularmente pelo insuficiente suprimento de nitrogênio (N) e fósforo (P) (ARAUJO et al., 2012).

Pode-se afirmar, que o crescimento da produção e o aumento da capacidade do feijão-caupi estão diretamente ligados com os avanços científicos e à disponibilização de tecnologias ao setor produtivo. Normalmente, para se alcançar elevada produtividade na cultura do feijão-caupi utilizam-se fertilizantes minerais, aumentando os custos de produção e ocasionando maior impacto ambiental. Após a descoberta do processo de fixação biológica de nitrogêno (FBN) com estirpes responsivas associadas à seleção de cultivares susceptíveis as interações, o feijão-caupi no Brasil vem dispensando a adubação mineral. No entanto, o gasto energético desprovido pelas bactérias neste processo é elevado, podendo levar a bactéria a não realizar este processo quando estiver presente o $\mathrm{N}$ mineral no solo, devido à redução da afinidade da leghemoglobina pelo oxigênio (DENISON; HARTER, 1995). Desta forma, buscam-se novas formas de melhorar a ef iciência da utilização do N pela cultura do feijão-caupi. Salienta-se que, a eficiência do processo de fixação de nitrogênio é dependente da disponibilidade de $P$ devido a sua participação no processo simbiótico (BURITY et al., 2000).

As novas pesquisas em FBN são realizadas com diferentes bactérias em diversas culturas, porém, poucos são os trabalhos que tentam associar dois gêneros diferentes de rizobactérias em cultivos de feijão-caupi. Neste contexto, bactérias diazotróficas do gênero Azospirillum merecem atenção, pois além da FBN, podem produzir compostos promotores de crescimento ou estimular a produção endógena da planta 
desses compostos (RODRIGUES et al., 2012). Diante do exposto, o presente trabalho teve por objetivo avaliar os efeitos da inoculação com Bradyrhizobium sp. e Azospirillum brasilense em associação com a adubação fosfatada sobre os componentes morfológicos e de produção da cultura do feijão-caupi cultivada na região sul do Estado de Rondônia.

\section{MATERIAL E MÉTODOS}

O experimento foi realizado no período de fevereiro a maio de 2018, em área experimental, munícipio de Colorado do Oeste, RO, cujas coordenadas geográficas são $13^{\circ} 06^{\prime} 23,51^{\prime \prime} \mathrm{S}$ e $60^{\circ} 30^{\prime} 37,65^{\prime \prime} \mathrm{W}$, com altitude média de 412 metros, em solo tipo Latossolo Vermelho-amarelo. O clima segundo a classificação de Köppen é do tipo Awa, tropical quente e úmido com duas estações bem definidas. Dados médios de temperatura e precipitação pluviométrica durante a condução do experimento foram obtidos do banco de dados do Instituto Nacional de Meteorologia (INMET).

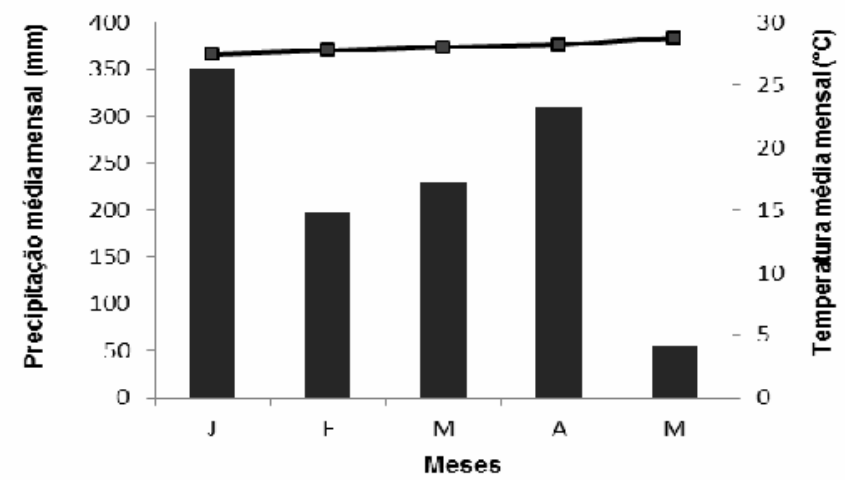

FIGURA 1- Precipitação $(\mathrm{mm})$ e temperatura $\left({ }^{\circ} \mathrm{C}\right)$ médias mensais, registradas na estação metereológica do Instituto Nacional de Meteorologia, no período de fevereiro a maio do ano agrícola de 2018.

A análise química da amostra de solo, na profundidade de $0-20 \mathrm{~cm}$, realizada na área experimental antes da instalação do experimento resultou nos seguintes valores: M.O.: $35,8 \mathrm{~g} \mathrm{dm}^{-3} ; \mathrm{pH}\left(\mathrm{CaCl}_{2}\right): 5,1 ; \mathrm{P}: 1 \mathrm{mg} \mathrm{dm}^{-3} ; \mathrm{K}: 2 \mathrm{mmolc} \mathrm{dm}{ }^{-3}$; Ca: $94 \mathrm{mmolc} \mathrm{dm}{ }^{-3}$; Mg: 13 mmolc dm ${ }^{-3}$; Al: 1 mmolc dm ${ }^{-3}$; $\mathrm{H}+\mathrm{Al}: 29$ mmolc dm${ }^{-3}$; SB: $109 \mathrm{mmolc} \mathrm{dm}^{-3}$; CTC: $138 \mathrm{mmolc} \mathrm{dm}^{-3}$, saturação por bases $79 \%$. A análise granulométrica apresentou $576 \mathrm{~g}$ $\mathrm{dm}^{-3}$ de argila, $178 \mathrm{~g} \mathrm{dm}^{-3}$ de areia e $246 \mathrm{~g} \mathrm{dm}^{-3}$ de silte.

$O$ delineamento experimental utilizado foi 0 de blocos casualizados, com três repetições, arranjados em esquema fatorial $3 \times 3$. O primeiro fator foi constituído por três doses de fósforo $\left(0,80\right.$ e $120 \mathrm{~kg} \mathrm{ha}^{-1}$ de $\left.\mathrm{P}_{2} \mathrm{O}_{5}\right)$; e o segundo fator, compreendeu ausência, inoculação e coinoculação com rizobactérias, perfazendo um total de 9 tratamentos, e 27 unidades experimentais. As doses de $\mathrm{P}$ foram aplicadas no sulco de plantio e a fonte utilizada foi o superfosfato simple $\left(21 \% \mathrm{P}_{2} \mathrm{O}_{5}\right)$. O genótipo de feijão-caupi utilizado foi o "roxinho da praia" com hábito ereto agudo, crescimento indeterminado e sem tendência a enrolar-se ao tutor. A adubação de base constou-se de nitrogênio e potássio, feita com a utilização de $30 \mathrm{~kg} \mathrm{ha}^{-1}$ de $\mathrm{Ne} 60 \mathrm{~kg} \mathrm{ha}^{-1}$ de $\mathrm{K}_{2} \mathrm{O}$, na forma de ureia $(45 \%$ de $\mathrm{N}$ ) e cloreto de potássio $\left(60 \%\right.$ de $\left.\mathrm{K}_{2} \mathrm{O}\right)$, respectivamente, incorporados. 
A inoculação das sementes de feijão-caupi, foi feita com inoculante comercial Total Nitro feijão-caupi (concentração de $2 \times 10^{9} \mathrm{UFC} / \mathrm{mL}$ ) da estirpe de Bradyrhizobium $s p$. (Semia 6462 e Semia 6463). A dose utilizada foi de $100 \mathrm{~mL}$ do inoculante liquido para 50 kg de sementes; e para a coinoculação foi utilizado o produto comercial contendo uma combinação de estirpes de Azospirillum brasilense. A dose aplicada foi de $150 \mathrm{~mL}$ para cada $50 \mathrm{~kg}$ de sementes. Ambos os inoculantes utilizados foram na formulação líquida, produzidos e cedidos pela Empresa Total Biotecnologia.

O preparo do solo incluiu a gradagem (grade de discos) até $15 \mathrm{~cm}$ de profundidade. Os sulcos de plantio e de adubação foram abertos manualmente com auxílio de enxada em profundidades entre 5 e $10 \mathrm{~cm}$. A semeadura foi realizada manualmente, com o auxílio de "matraca", colocando-se quatro sementes por cova, deixando-se após o desbaste cinco plantas por metro linear. Cada unidade experimental foi composta por 3 linhas de 4 metros de comprimento, espaçadas em 0,60 m entre linhas e 0,30 m entre plantas. Consideraram-se como parcela útil a linha central, excluindo-se $0,5 \mathrm{~m}$ de cada extremidade da parcela. Aos oito dias após a emergência (DAE) das plantas realizou-se o desbaste, deixando-se apenas uma planta/cova. Aos 10 e 30 DAE das plantas procedera a aplicação de inseticida com ingrediente ativo Imidacloprido $100 \mathrm{~g} / \mathrm{L}$, Beta-ciflutrina 12,5 g/L, grupo químico Neonicotinoide (Imidacloprido) e Piretroide (Beta-ciflutrina), com recomendação para a cultura do feijão-caupi de 750-1000 mL/ha, para controle de Diabrotica speciosa. As capinas foram realizadas mensalmente para controle de plantas daninhas.

Por ocasião do pleno florescimento ( $50 \%$ das plantas em floração) da cultura coletou-se de 20 folhas trifolioladas por parcela para a determinação dos teores foliares de macronutrientes. A amostragem foi feita no terço mediano das plantas da área útil das parcelas. Todo o material vegetal coletado foi lavado em água corrente e água deionizada; e as amostras foram acondicionadas em sacos de papel e secos em estufa com circulação forçada de ar, à temperatura de $65^{\circ} \mathrm{C}$, por 72 horas, sendo posteriormente moídas. As amostras moídas foram submetidas à digestão sulfúrica e digestão nitroperclórica, utilizando a metodologia descrita em Embrapa (2009). Foi determinada a altura das plantas, obtida pela medição do colo da planta até o meristema apical, utilizando-se de fita métrica; o diâmetro do caule, determinado com o auxílio de paquímetro digital, na altura de $2 \mathrm{~cm}$ do colo da planta; o número de folhas por plantas e o número de nódulos viáveis, obtido a partir da contagem; enquanto que as avaliações do número de vagens por planta, número de grãos por vagem e número de grãos por planta foram determinados a partir do número médio de vagens colhidas em cinco plantas da área útil da parcela; e a produção de massa seca da parte aérea, massa seca da raiz e produtividade de grãos foram determinadas no período de maturação fisiológica da cultura, estádio fenológico R5. A produtividade foi obtida pelo peso de grãos da área útil em quilogramas, com correção para $13 \%$ de umidade, transformando os dados para $\mathrm{kg} \mathrm{ha}^{1}$.

Os dados foram submetidos ao teste de normalidade (Shapiro Wilk) e análise de variância pelo teste de Tukey a nível de 5\% de probabilidade, com auxílio computacional do programa de análise estatística Sisvar.

\section{RESULTADOS E DISCUSSÃO}

De acordo com análise de variância, os resultados evidenciaram efeitos significativos $(p \leq 0,05)$ da interação bactéria $(B)$ e dose de fósforo (D) para altura de plantas, diâmetro do caule, número de folhas, número de nódulos, número de vagem por planta, comprimento de vagem, largura de vagem, número de sementes 
por vagem, número de sementes por planta, massa seca da parte aérea, massa seca da raiz e produtividade de plantas de feijão-caupi; enquanto que as concentrações de $\mathrm{N}$ e $\mathrm{K}$ nas folhas em pleno florescimento apresentaram efeito significativo $(p \leq 0,05)$ apenas para dose de fósforo, e as concentrações de $\mathrm{P}, \mathrm{Ca}, \mathrm{Mg}$ e $\mathrm{S}$ não foram influenciadas por nenhum dos fatores estudados.

$\mathrm{O}$ incremento nas doses de $\mathrm{P}$ proporcionou aumento na altura de plantas, diâmetro do caule e número de folhas de plantas de feijão caupi, diferindo estatisticamente $(p \leq 0,05)$ do tratamento controle $\left(0 \mathrm{~kg}\right.$ ha ${ }^{-1}$ de $\mathrm{P}_{2} \mathrm{O}_{5}$ ) (Tabela 1), evidenciando que a ausência de fósforo é um fator limitante ao crescimento e ao desenvolvimento de plantas de feijãocaupi, comprometendo os componentes morfológicos da planta. No desdobramento da interação bactéria e doses de $\mathrm{P}$, a inoculação de Bradyrhizobium sp. acrescida de $120 \mathrm{~kg}$ ha ${ }^{-1}$ de $\mathrm{P}_{2} \mathrm{O}_{5}$ promoveu aumento na altura de plantas, no diâmetro do caule e no número de folhas de plantas de feijão-caupi, na ordem de 18,36\%, 63,75\% e 42,86\%, respectivamente, em relação ao tratamento inoculado e sem adubação fosfatada. Quando comparado com o controle absoluto (sem inoculação e sem adubação), esse aumento foi de $53,31 \%$, superior a $100 \%$ e $77,44 \%$, respectivamente, não diferindo estatisticamente do tratamento coinoculado (Tabela 1). Essa análise da altura é importante na avaliação da qualidade de plantas, uma vez que fornece um bom indicador de evolução da cultura (SOUTO et al., 2009); enquanto que o efeito do $P$ sobre o diâmetro do caule, indica relação do nutriente com o aumento da resistência da planta ao acamamento (OLIVEIRA et al., 2011).

O número de nódulos viáveis aumentou significativamente $(\mathrm{p} \leq 0,05)$ com o aumento das doses de fósforo e na presença de inoculação com Bradyrhizobium sp., diferindo estatisticamente $(p \leq 0,05)$ do controle absoluto (sem inoculação e sem adubação) e do tratamento coinoculado (Bradyrhizobium sp. + Azospirillum brasilense) (Tabela 1). Esses dados corroboram aos encontrados por Araujo et al. (2018) em solos amazônicos. O efeito positivo da inoculação de Bradyrhizobium sp. quando associada à adubação fosfatada, auxilia na simbiose, pois quando se aplica este nutriente há maior formação de nódulos na raiz, visto que o $\mathrm{P}$ é fonte de energia para planta que quando bem nutrida, disponibiliza carboidratos para as bactérias de rizóobio, auxiliando no desenvolvimento de nódulos. Além disso, a inoculação na presença de adubação fosfatada apresentava capacidade de formar nódulos suficientes para garantir a fixação biológica de nitrogênio.

A produção de massa seca da parte aérea e massa seca da raiz foram incrementadas com as doses de $\mathrm{P}$, demonstrando que a dose de $120 \mathrm{~kg} \mathrm{ha}^{-1}$ de $\mathrm{P}_{2} \mathrm{O}_{5}$ foi a que promoveu maior produção de massa seca da parte aérea e da raiz (Tabela 1). No desdobramento da interação, a dose de $120 \mathrm{~kg}^{-1}$ de $\mathrm{P}_{2} \mathrm{O}_{5}$, na presença de Bradyrhizobium sp. proporcionou incremento superior a $100 \%$, na produção de massa seca da parte aérea e massa seca da raiz, quando comparado ao controle absoluto (sem inoculação e sem adubação), não diferindo estatisticamente do tratamento coinoculado (Bradyrhizobium sp. + Azospirillum brasilense) e adubado com $120 \mathrm{~kg} \mathrm{ha}^{-1}$ de $\mathrm{P}_{2} \mathrm{O}_{5}$. (Tabela 1). Esses dados confirmam o sinergismo entre a adubação fosfatada e a inoculação com rizobactérias em plantas de feijão-caupi. Isso pode ser explicado porque o fósforo auxilia no aumento do crescimento dos pêlos radiculares, que por sua vez são locais de infecção das bactérias, então, em ambiente com pouca disponibilidade de $\mathrm{P}$, a bactéria tem sua eficiência limitada, pela falta de locais de infecção, situação que é resolvida quando se fornece doses adequadas de $\mathrm{P}$.

Os parâmetros fitotécnicos de número, comprimento e largura de vagens, bem como, a produção de grãos por vagem e a produção de grãos por planta 
foram significativamente $(p \leq 0,05)$ influenciados pela interação bactéria e doses de fósforo. A dose de $120 \mathrm{~kg} \mathrm{ha}^{-1}$ de $\mathrm{P}_{2} \mathrm{O}_{5}$, na presença de Bradyrhizobium sp. promoveu aumento de $33,33 \%$ no número de vagens, $14,95 \%$ no comprimento de vagens, $16,93 \%$ na largura de vagens, $5,68 \%$ na produção de grãos por vagem e $39 \%$ na produção de grãos por planta, quando comparado ao tratamento apenas inoculado com Bradyrhizobium sp. A maior emissão e crescimento de folhas e maior área foliar, contribui para maior captação de radiação solar e incremento na produção de fotoassimilados, podendo aumentar na relação matéria seca da parte aérea e raiz, assim como proporcionar aumento no número de vagens, número de grãos e massa de grãos, principais determinantes da produtividade de plantas. Zucarelli et al., (2011), relataram que o maior número de vagens por planta influenciado pelas doses de $\mathrm{P}$, possivelmente, deve-se ao $\mathrm{P}$ estimular o desenvolvimento radicular, favorecendo a formação dos primórdios das partes reprodutivas, assim como proporciona às plantas maior resistência às adversidades, o que possibilita condições para o feijão-caupi produzir maior quantidade de sementes e de melhor qualidade, uma vez que este nutriente é componente da fitina, principal forma de armazenamento de fósforo na semente (GRANT et al., 2001).

A máxima produtividade de grãos de feijão-caupi também foi obtida com a aplicação de $120 \mathrm{~kg} \mathrm{ha}^{-1}$ de $\mathrm{P}_{2} \mathrm{O}_{5}$ na presença de Bradyrhizobium sp., não diferindo estatisticamente $(p \leq 0,05)$ do tratamento coinoculado e adubado com $120 \mathrm{~kg} \mathrm{ha}^{-1} \mathrm{de}_{2} \mathrm{P}_{5}$. A média de produtividade quando inoculado e coinoculado na presença de $120 \mathrm{~kg} \mathrm{ha}^{-1} \mathrm{de}$ $\mathrm{P}_{2} \mathrm{O}_{5}$ foi de $883 \mathrm{~kg} \mathrm{ha}^{-1}$; enquanto que a média de produtividade do tratamento inoculado e coinoculado na ausência de adubação fosfatada foram de $636 \mathrm{~kg} \mathrm{ha}^{-1}$, com incrementos na ordem de $38 \%$ quando comparados. Pode-se relatar que o incremento em produtividade é alcançado somente com o suprimento de fósforo em quantidades compatíveis com a demanda da cultura, valendo salientar que essa resposta está diretamente relacionada com os níveis de $\mathrm{P}$ existentes no solo. No presente estudo o teor de $\mathrm{P}$ no solo encontrava-se com $1 \mathrm{mg} \mathrm{dm}^{-3}$, teor considerado muito baixo, o que justifica a resposta dos parâmetros morfológicos e de produção em função das doses do nutriente. Contudo, é possível afirmar que a inoculação de Bradyrhizobium sp. associada a adubação fosfatada de $120 \mathrm{~kg} \mathrm{ha}^{-1}$ de $\mathrm{P}_{2} \mathrm{O}_{5}$ é suficiente para promover o máximo crescimento e desenvolvimento de plantas de feijão-caupi cultivadas em solos amazônicos. 
TABELA 1- Parametros fitotécnicos da cultura do feijão-caupi, genótipo roxinho da praia, inoculados e coinoculados com rizobactérias em associação com adubação fosfatada. Colorado do Oeste, RO (2018).

\begin{tabular}{|c|c|c|c|c|c|c|}
\hline \multirow[b]{2}{*}{ Bactéria } & \multicolumn{6}{|c|}{ Doses de $\mathrm{P}_{2} \mathrm{O}_{5}\left(\mathrm{~kg} \mathrm{ha}^{-1}\right)$} \\
\hline & 0 & 80 & 120 & 0 & 80 & 120 \\
\hline & \multicolumn{3}{|c|}{ Atura de plantas (cm) } & \multicolumn{3}{|c|}{ Diâmetro do caule (mm) } \\
\hline SI & $75,66 \mathrm{cB}$ & $96,00 \mathrm{bA}$ & $106,33 \mathrm{aB}$ & $8,42 \mathrm{cC}$ & $12,08 \mathrm{bB}$ & $17,65 \mathrm{aB}$ \\
\hline I & $98,00 \mathrm{bA}$ & $99,33 \mathrm{bA}$ & $116,00 \mathrm{aA}$ & $12,80 \mathrm{cA}$ & $18,25 \mathrm{bA}$ & 20,96 aA \\
\hline \multirow[t]{2}{*}{$\mathrm{CO}$} & $96,66 \mathrm{bA}$ & $96,00 \mathrm{bA}$ & $111,00 \mathrm{aA}$ & $13,38 \mathrm{cA}$ & $18,28 \mathrm{bA}$ & $21,20 \mathrm{aA}$ \\
\hline & \multicolumn{3}{|c|}{ Número de folhas (un.) } & \multicolumn{3}{|c|}{ Número de nódulos (un.) } \\
\hline SI & $20,66 \mathrm{cB}$ & $28,00 \mathrm{bA}$ & $37,00 \mathrm{aA}$ & $3,33 \mathrm{cC}$ & $11,00 \mathrm{bC}$ & $22,66 \mathrm{aB}$ \\
\hline 1 & $25,66 \mathrm{bA}$ & $28,66 \mathrm{bA}$ & $36,66 \mathrm{aA}$ & $20,33 \mathrm{bA}$ & $20,66 \mathrm{bA}$ & $41,66 \mathrm{aA}$ \\
\hline \multirow[t]{2}{*}{$\mathrm{CO}$} & $21,00 \mathrm{bB}$ & $31,00 \mathrm{aA}$ & 34,33 aA & $12,30 \mathrm{cB}$ & $18,66 \mathrm{bB}$ & $21,00 \mathrm{aB}$ \\
\hline & \multicolumn{3}{|c|}{ Massa seca parte aérea (g) } & \multicolumn{3}{|c|}{ Massa seca da raiz $(\mathrm{g})$} \\
\hline $\mathrm{SI}$ & $41,97 \mathrm{cB}$ & $58,71 \mathrm{bB}$ & $70,32 \mathrm{aB}$ & $3,66 \mathrm{cB}$ & $8,09 \mathrm{bC}$ & $10,33 \mathrm{aB}$ \\
\hline I & $63,30 \mathrm{cA}$ & $72,43 \mathrm{bA}$ & 86,14 aA & $5,33 \mathrm{cA}$ & $12,96 \mathrm{bA}$ & 14,57 aA \\
\hline \multirow[t]{2}{*}{$\mathrm{CO}$} & $67,47 \mathrm{cA}$ & $72,19 \mathrm{bA}$ & 86,27 aA & $5,21 \mathrm{cA}$ & $10,89 \mathrm{bB}$ & $13,91 \mathrm{aA}$ \\
\hline & \multicolumn{3}{|c|}{ Número de vagens (un.) } & \multicolumn{3}{|c|}{ Comprimento de vagem (cm) } \\
\hline SI & $0,00 \mathrm{cB}$ & $3,00 \mathrm{bA}$ & $4,00 \mathrm{aA}$ & $0,00 \mathrm{cB}$ & $20,96 \mathrm{bA}$ & $22,22 \mathrm{aB}$ \\
\hline I & $3,00 \mathrm{bA}$ & $3,00 \mathrm{bA}$ & $4,00 \mathrm{aA}$ & $21,47 \mathrm{bA}$ & $21,75 \mathrm{bA}$ & $24,68 \mathrm{aA}$ \\
\hline $\mathrm{CO}$ & $3,00 \mathrm{bA}$ & $3,00 \mathrm{bA}$ & 3,66 aA & 20,89 bA & $21,06 \mathrm{bA}$ & $23,22 \mathrm{aB}$ \\
\hline
\end{tabular}




\begin{tabular}{|c|c|c|c|c|c|c|}
\hline & \multicolumn{3}{|c|}{ Largura da vagem (mm) } & \multicolumn{3}{|c|}{ Número de sementes/vagem (un.) } \\
\hline $\mathrm{SI}$ & $0,00 \mathrm{cB}$ & $10,53 \mathrm{bA}$ & $11,89 \mathrm{aA}$ & $0,00 \mathrm{cB}$ & $14,00 \mathrm{bB}$ & $16,00 \mathrm{aA}$ \\
\hline I & $9,98 \mathrm{bA}$ & 11,19 aA & $11,67 \mathrm{aA}$ & $15,66 \mathrm{bA}$ & 16,66 aA & $16,55 \mathrm{aA}$ \\
\hline \multirow[t]{2}{*}{$\mathrm{CO}$} & $10,67 \mathrm{bA}$ & 11,10 aA & $11,48 \mathrm{aA}$ & $15,66 \mathrm{aA}$ & 15,66 aA & $16,00 \mathrm{aA}$ \\
\hline & \multicolumn{3}{|c|}{ Número de sementes/ planta (un.) } & \multicolumn{3}{|c|}{ Produtividade $\left(\mathrm{kg} \mathrm{ha}^{-1}\right)$} \\
\hline $\mathrm{SI}$ & $0,00 \mathrm{cB}$ & $42,00 \mathrm{bB}$ & $58,66 \mathrm{aB}$ & $0,00 \mathrm{cB}$ & $567,00 \mathrm{bB}$ & $792,00 \mathrm{aB}$ \\
\hline I & $\begin{array}{l}47,00 \mathrm{bA} \\
47,00 \mathrm{bA}\end{array}$ & $\begin{array}{l}50,00 \mathrm{bA} \\
47,00 \mathrm{bA}\end{array}$ & $\begin{array}{l}65,33 \mathrm{aA} \\
64,00 \mathrm{aA}\end{array}$ & $\begin{array}{l}637,50 \mathrm{bA} \\
634,50 \mathrm{bA}\end{array}$ & $\begin{array}{l}675,00 \mathrm{bA} \\
634,50 \mathrm{bA}\end{array}$ & $\begin{array}{l}882,00 \mathrm{aA} \\
864,00 \mathrm{aA}\end{array}$ \\
\hline
\end{tabular}

As letras minúsculas separam as médias dentro de cada coluna para o fator dose de $\mathrm{P}$ e as letras maiúsculas separam as médias dentro da linha para o fator bactéria. Letras iguais não diferem entre si pelo teste de Tukey a $5 \%$ de probabilidade. SI: sem inoculação; I: inoculado com Bradyrhizobium sp.; CO: coinoculado com Bradyrhizobium sp + Azospirillum brasilense 
O feijoeiro apresenta como característica baixa exigência em $P$, contudo tem apresentado maior e frequente resposta quando cultivado em solo com boa disponibilidade do nutriente (ARAUJO et al., 2018). Os teores de $\mathrm{N}$ e K nas folhas de feijão-caupi em pleno florescimento responderam significativamente $(p \leq 0,05)$ a adição de $\mathrm{P}$, sendo a máxima concentração de $\mathrm{N}$ e K nas folhas de $36,66 \mathrm{~g} \mathrm{~kg}^{-1} \mathrm{e}$ $19,68 \mathrm{~g} \mathrm{~kg}^{-1}$, repectivamente, obtida na dose $80 \mathrm{~kg} \mathrm{ha}^{-1}$ de $\mathrm{P}_{2} \mathrm{O}_{5}$, não apresentando diferença significativa da dose de $120 \mathrm{~kg} \mathrm{ha}^{-1}$ de $\mathrm{P}_{2} \mathrm{O}_{5}$ (Figura 2A e 2B). No entanto, embora não tenha sido constatada diferença significativa entre as doses de 80 e 120 $\mathrm{kg} \mathrm{ha}^{-1}$ de $\mathrm{P}_{2} \mathrm{O}_{5}$, é possível observar ligeira redução dos teores de nutrientes na dose mais elevada. Esse aumento nos teores foliares de macronutrientes com a aplicação da adubação fosfatada pode explicar um futuro aumento da produção de grãos, considerando-se que há relatos na literatura a respeito da estreita correlação entre teores foliares de nutrientes e produtividade das culturas (ARAÚJO et al., 2017).
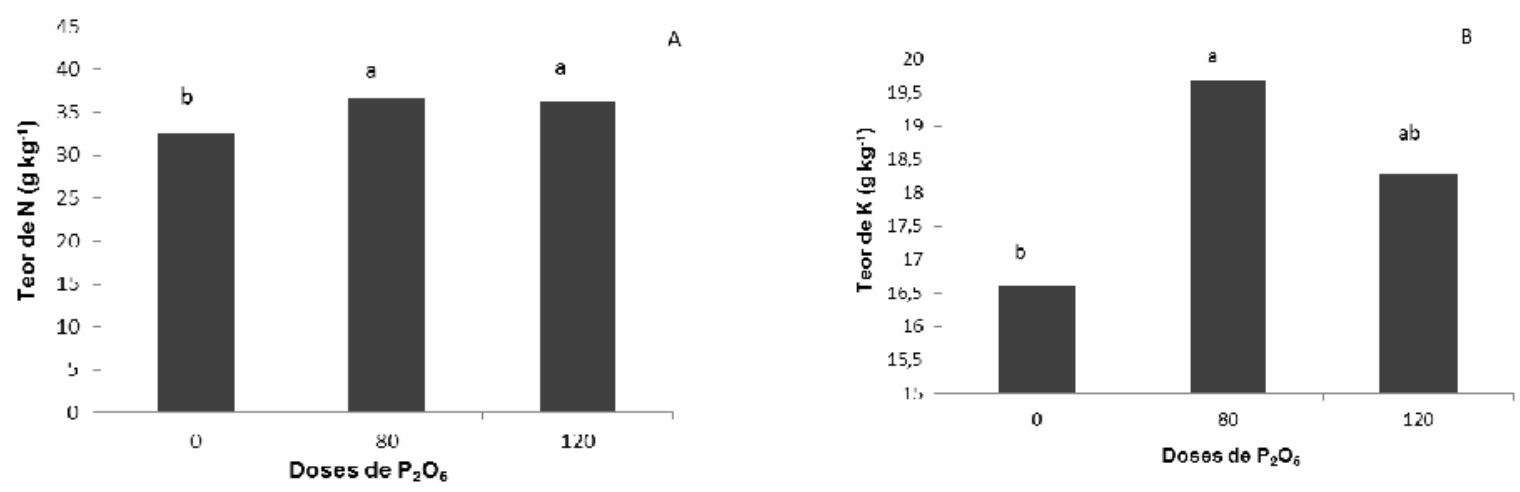

FIGURA 2- Teor de N e K nas folhas de feijão-caupi em resposta a adubação fosfatada.

\section{CONCLUSÕES}

A dose de $120 \mathrm{~kg} \mathrm{ha}^{-1}$ de $\mathrm{P}_{2} \mathrm{O}_{5}$, na presença de Bradyrhizobium sp. é suficiente para promover o máximo crescimento e desenvolvimento de plantas de feijão-caupi cultivada em ambiente amazônico.

A produtividade de grãos aumentou na ordem de $38 \%$ quando inoculado com Bradyrhizobium sp. e adubado com $120 \mathrm{~kg} \mathrm{ha}^{-1}$ de $\mathrm{P}_{2} \mathrm{O}_{5}$.

A adição de $\mathrm{P}_{2} \mathrm{O}_{5}$ no solo promoveu aumento nas concentrações de $\mathrm{N}$ e $\mathrm{K}$ nas folhas de plantas de feijão-caupi em pleno florescimento.

A coinoculação (Bradyrhizobium sp. + Azospirillum brasilense) não influenciou os componentes morfológicos e produtivos da cultura do feijão-caupi, no entanto deve ser mais bem elucidada, visto que há possibilidades de interação entre os microrganismos, contribuindo para o alcance da sustentabilidade no setor agrícola.

\section{AGRADECIMENTOS}

O autor agradece à Fundação de Estudos Agrários Luiz de Queiroz - FEALQ e a Empresa Total Biotecnologia pela disponibilização de recursos. 


\section{REFERÊNCIAS}

ARAÚJO, E.O.; MAUAD, M.; TADEU, H.C.; LIMA FILHO, H.A.; SILVA,, J.A.F.; CARDOSO, J.A. Nutritional status of cowpea plants inoculated with Bradyrhizobium and Azospirillum brasilense in associated with phosphate fertilization in soil Amazonian. Journal of Experimental Agriculture International, v.23, n.5, p.1-13, 2018. Disponivel em: http://www.sciencedomain.org/review-history/24992

ARAUJO, E.O.; GEROLA, J.G.; FERREIRA, C.R.G.; MATTE, L.C. Effect of rates and sources of soluble phosphorus on the behavior of cowpea plants inoculated with rhizobacteria from soils of the north region of Brazil. African Journal Microbiology Research, v.11, n.42, p. 1544-1550, 2017. Disponivel em: https://doi.org/10.5897/AJMR2017.8649

ARAUJO, E.O., SANTOS, E.F., CAMACHO, M.A. Nutritional efficiency of cowpea varieties in the absorption of phosphorus. Agronomía Colombiana, n.30, v.3, p. 419-424, 2012. Disponivel em: http://www.scielo.org.co/pdf/agc/v30n3/v30n3a14.pdf

BURITY, H. A.; LYRA, M. C. C. P.; SOUZA, E. S. Efetividade da inoculação com rizóbio e fungosmicorrízicosarbusculares em mudas de sabiá submetidas a diferentes níveis de fósforo. Pesquisa Agropecuária Brasileira, Brasília, v. 35, p. 801-807, 2000. Disponivel em: http://dx.doi.org/10.1590/S0100204X2000000400018.

CONAB. 2018. Acompanhamento da Safra Brasileira de Grãos 2017/2018: Nono Levantamento, Junho/2018, Companhia Nacional de Abastecimento. Brasília, CONAB, 178p.

COUTINHO, W.R.; DA SILVA, D.M.S.; SALDANHA, E.C.M.; OKUMURA, R.S.; SILVA JÚNIOR, M.L. Doses de fósforo na cultura do feijão-caupi na região nordeste do estado do Pará. Revista Agro@mbiente On-line, v. 8, n. 1, p. 66-73, 2014. Disponivel em: https://www.researchgate.net/publication/290460468_Doses_de_fosforo_na_cultura_ do_feijao-caupi_na_regiao_nordeste_do_Estado_do_Para

DENISON, R.F.; HARTER, B.L. Nitrate effects on nodule oxygen permeability and leghemoglobin: Nodule oximetry and computer modeling. Plant Physiology, v. 107, p. 1355-1364, 1995. Disponivel em: https://doi.org/10.1104/pp.107.4.1355

EMPRESA BRASILEIRA DE PESQUISA AGROPECUARIA - EMBRAPA. Manual de análises químicas de solos, plantas e fertilizantes. - 2. Ed, Brasília., Embrapa Informação Tecnológica, 2009. 627 p.

GRANT, C. A.; FLATEN, D. N.; TOMASIEWICZ, D. J.; SHEPPARD, S. C. The importance of early season phosphorus nutrition. Canadian Journal of Plant Sciences, v. 81, n. 2, p. 211-224, 2001. Disponivel em: https://doi.org/10.4141/P00093

OLIVEIRA, G. A.; ARAÚJO, W. F.; CRUZ, P. L. S.; SILVA, W. L. M.; FERREIRA, G. B. Resposta do feijão-caupi as lâminas de 
irrigação e as doses de fósforo no cerrado de Roraima. Revista Ciência Agronômica, v. 42, n. 4, p. 872-882, 2011. Disponivel em: http://ccarevista.ufc.br/seer/index.php/ccarevista/article/view/1178/621

RODRIGUES, A.C.; ANTUNES, J.E.L.; MEDEIROS, B.G.F.; BARROS, M.V.B. Resposta da co-inoculação de bactérias promotoras de crescimento em plantas e Bradyrhizobium sp. em caupi. Bioscience Journal, v.28, p. 196-202, 2012. Disponivel em: http://ccarevista.ufc.br/seer/index.php/ccarevista/article/view/1178/621

SILVA, R. P.; SANTOS, C. E.; LIRA JÚNIOR, M. A.; STAMFORD, N. P. Efetividade de estirpes selecionadas para feijão caupi em solo da região semi-árida do sertão da Paraíba. Revista Brasileira de Ciências Agrárias, v. 3, n. 2, p. 105-110, 2008. Disponivel em: http://132.248.9.34/hevila/AgrariaRecife/2008/vol3/no2/1.pdf

SOUTO, J. S.; OLIVEIRA, F. T.; GOMES, M. M. S.; NASCIMENTO, J. P.; SOUTO, P. C. Efeito da aplicação de fósforo no desenvolvimento de plantas de feijão guandu Cajanus cajan (L) Millsp). Revista Verde, v. 4, n. 1, p. 135 - 140, 2009. Disponivel em : https://www.gvaa.com.br/revista/index.php/RVADS/article/viewFile/160/160

ZUCARELI, C.; PRANDO, A. M.; RAMOS JUNIOR, E. U.; NAKAGAWA, J. Fósforo na produtividade e qualidade de sementes de feijão carioca precoce cultivado no período das águas. Revista Ciência Agronômica, v. 42, n. 1, p. 32-38, 2011. Disponivel em: http://ccarevista.ufc.br/seer/index.php/ccarevista/article/view/749 